\title{
Numerical Study of Spurious Inertial Modes in Shallow Water Models for a Variable Bathymetry
}

\author{
Gossouhon SITIONON ${ }^{1}$, Adama COULIBALY ${ }^{1}$, Jérôme Kablan ADOU \\ ${ }^{1}$ Université Félix Houphouet Boigny, Abidjan, 22 BP 582 Abidjan 22, UFR Mathématiques et Informatique, Côte d'Ivoire \\ Correspondence: Gossouhon SITIONON, Université Félix Houphouet Boigny, Abidjan, 22 BP 582 Abidjan 22, UFR \\ Mathématiques et Informatique, Côte d'Ivoire. E-mail: gossouhon.sitionon@gmail.com
}

Received: September 16, 2019

doi:10.5539/jmr.v11n6p58
Accepted: October 23, 2019 Online Published: November 13, 2019

URL: https://doi.org/10.5539/jmr.v11n6p58

\begin{abstract}
In this study we perform a modal analysis of the linear inviscid shallow water equations using a non constant bathymetry, continuous and discontinuous Galerkin approximations. By extracting the discrete eigenvalues of the resulting algebraic linear system written on the form of a generalized eigenvalue / eigenvector problem we first show that the regular variation of the bathymetry does not prevent the presence of spurious inertial modes when centered finite element pairs are used. Secondly, we show that such spurious modes are not present in discontinuous Galerkin discretizations when all variables are approximated in the same descrete space. Such spurious inertial modes have been found very damageable for the quality of inertia-gravity and Rossby modes in ocean modelling.
\end{abstract}

Keywords: discontinuous Galerkin, mixed finite element, shallow water equations, spurious modes, variable bathymetry

Mathematics Subject Classification: 65M60; 76M10

\section{Introduction}

For most discretization schemes, the numerical approximation of shallow water models for geophysical flows is a delicate problem. See ( Eldred C. \& Le Roux D.Y.(2018), Eldred C. \& Le Roux D.Y.(2019)). Indeed, the coupling between the continuity and momentum equations usually produces non physical oscillations in the solution, called spurious oscillations. Numerical study of the shallow water equations for finite element pairs that have two velocity components per node brings out three kind of modes namely the physical geostrophic and inertia-gravity modes and non physical spurious inertial modes which appear when the number of discrete momentum equations exceeds, at least by a factor of two, the number of discrete continuity equations. Existence and proportions of theses modes can be found in some papers such as ( Le ROUX, 2012) but their authors have only considered the case of constant bathymetry. Indeed, it has been shown that the spurious inertial modes have the frequency $\pm f$, when the Coriolis factor $f$ and the bathymetry $H$ are constant, by using Fourier analysis. Such a Fourier study is not possible when the bathymetry $H(x, y)$ is no longer constant. The first aim of this paper is to examine numerically the existence and proportions among all the discrete frequencies, of the spurious inertial modes for five finite element pairs in the case of a variable bathymetry and compare the results obtained with those of constant bathymetry. The second aim of this paper is to find a method that makes velocity and surface elevation free of spurious inertial modes, namely, the discontinuous Galerkin method.

We first present the model and perform the temporal and space discretizations. The continuous and discontinuous Galerkin formulations are then presented and concluding remarks complete the study.

\section{Governing Equations}

In this study we focus on the inviscid shallow water (SW) equations linearized around a state of rest, with a variable bathymetry $H(x, y)$ and a constant Coriolis force $f$. Let $\Omega$ be the domain and $\Sigma$ its boundary. The linearized inviscid shallow water equations in cartesian coordinates are expressed as follows:

$$
\begin{aligned}
\widetilde{\mathbf{u}}_{t}+f \mathbf{k} \times \widetilde{\mathbf{u}}+g \nabla \widetilde{\eta} & =0, \\
\widetilde{\eta}_{t}+\nabla \cdot(H \widetilde{\mathbf{u}}) & =0,
\end{aligned}
$$

where $g$ is the gravitational acceleration, $\widetilde{\mathbf{u}}=(\widetilde{u}, \widetilde{v})$ is the velocity field vector, $\widetilde{\eta}$ is the surface elevation with respect to 
the reference level $z=0, \mathbf{k}$ is a unit vector in the vertical direction such that $\mathbf{k} \times \widetilde{\mathbf{u}}=(-\widetilde{v}, \widetilde{u})$ and let $\mathbf{x}=(x, y)$.

The system (1) -(2) is solved subject to periodic boundary condition to mimic the Fourier analyses of previous studies.

\section{Discretization of the Inviscid Linear Shallow-Water Equations}

\subsection{The Finite Element Method}

For a given time step $\Delta t=t_{n+1}-t_{n},\left(n=0 ; 1 ; 2 ; \ldots\right.$ and $\left.t_{0}=0\right)$, the time discretization of (1) - (2) is written using a Crank Nicolson scheme. See (Adou, Sitionon \& Coulibaly, 2016). By looking for periodic Fourier solutions such as

$$
\left(\widetilde{\mathbf{u}}^{n}\left(\mathbf{x}, t_{n}\right), \widetilde{\eta}^{n}\left(\mathbf{x}, t_{n}\right)\right)=(\mathbf{u}(\mathbf{x}), \eta(\mathbf{x})) e^{i \omega t_{n}},
$$

where $\mathbf{u}(\mathbf{x})$ and $\eta(\mathbf{x})$ are the amplitudes of the velocity field and the free surface elevation respectively, with $t_{n}=n \Delta t$. We let $\alpha=\frac{E-1}{\frac{\Delta t}{2}(E+1)}$ with $E=e^{i \omega \Delta t}$ and we obtain

$$
\begin{aligned}
\alpha \mathbf{u}+f \mathbf{k} \times \mathbf{u}+g \boldsymbol{\nabla} \eta & =0, \\
\alpha \eta+\nabla .(H \mathbf{u}) & =0 .
\end{aligned}
$$

To obtain the weak formulation either the gradient term in (4) or the divergence term in (5) is integrated by parts, which requires two different weak formulations, $\mathbf{F 1}$ and $\mathbf{F} 2$ respectively, defined as follow.

Formulation F1: Let $\mathbf{V}_{\mathbf{1}}$ be a subspace of $\left(H^{1}(\Omega)\right)^{2}, Q_{1}$ be a subspace of $L^{2}(\Omega), \mathbf{u} \in \mathbf{V}_{\mathbf{1}}$ and $\eta \in Q_{1}$. The weak formulation is obtained by multiplying (4) by a test function $\phi(\mathbf{x})$ and then multiplying (5) by a test function $\zeta(\mathbf{x})$, with $\phi(\mathbf{x}) \in \mathbf{V}_{\mathbf{1}}$ and $\zeta(\mathbf{x}) \in Q_{1}$, and integrating over the domain which leads to

$$
\begin{aligned}
\alpha \int_{\Omega} \mathbf{u} . \phi d \Omega+f \int_{\Omega}(\mathbf{k} \times \mathbf{u}) \cdot \phi d \Omega-g \int_{\Omega} \eta \nabla \cdot \phi d \Omega & =0, \\
\alpha \int_{\Omega} \eta \zeta d \Omega+\int_{\Omega} \nabla \cdot(H \mathbf{u}) \zeta d \Omega & =0 .
\end{aligned}
$$

Formulation F2: Let $\mathbf{u}$ belongs to $\mathbf{V}_{\mathbf{2}}$, a subspace of $\left(L^{2}(\Omega)\right)^{2}$ and let $\eta$ be in a subspace $Q_{2}$ of $H^{1}(\Omega)$. Equation (4) is multiplied by a test function $\varphi(\mathbf{x})$ with $\varphi(\mathbf{x}) \in \mathbf{V}_{2}$ and equation (5) is multiplyed by a test function $\psi(\mathbf{x})$ belonging to $Q_{2}$ and we integrate over the domain $\Omega$ to obtain

$$
\begin{aligned}
\alpha \int_{\Omega} \mathbf{u} \cdot \varphi d \Omega+f \int_{\Omega}(\mathbf{k} \times \mathbf{u}) \cdot \varphi d \Omega+g \int_{\Omega} \varphi \cdot \nabla \eta d \Omega & =0, \\
\alpha \int_{\Omega} \eta \psi d \Omega-\int_{\Omega} H \mathbf{u} \cdot \boldsymbol{\nabla} \psi d \Omega & =0 .
\end{aligned}
$$

The Galerkin method approaches the solutions of (6)-(9) in a finite dimensional subspace. Consider a triangulation $T_{h}$, of the polygonal domain $\Omega$, where $h$ is the parameter representative of the mesh length which measures the resolution. Let $\mathbf{V}_{j, h}$ and $Q_{j, h}$, be the finite-dimensional subspaces of $\mathbf{V}_{j}$ and $Q_{j}$, respectively, for $j=1,2$. The solution $\mathbf{u}_{h}$ (resp. $\eta_{h}$ ) belongs to $\mathbf{V}_{j, h}$ (resp. $\mathbf{Q}_{j, h}$ ) defined as beeing the set of functions $\mathbf{u}_{h}$ (resp. $\eta_{h}$ ) whose restriction on a triangle $K$ of $T_{h}$ belongs to $P_{m_{1}}(K) \times P_{m_{1}}(K)$ (resp. $P_{m_{2}}(K)$ ), where $P_{m_{1}}(K)$ ( resp. $\left.P_{m_{2}}(K)\right)$ denotes the set of polynomials of degree $m_{1}$ (resp. $m_{2}$ ) defined on $K$.

The introduction of the finite element basis leads to a formulation as in (6)-(9), but with $\mathbf{u}, \eta$ replaced by the finite element trial functions $\mathbf{u}_{h}, \eta_{h}$ and $\boldsymbol{\phi}, \boldsymbol{\varphi}, \zeta, \psi$ replaced by the corresponding finite element test functions $\boldsymbol{\phi}_{h} \in \mathbf{V}_{1, h}, \boldsymbol{\varphi}_{h} \in \mathbf{V}_{2, h}$, $\zeta_{h} \in Q_{1, h}$ and $\psi_{h} \in Q_{2, h}$. Further, the solutions $\mathbf{u}_{h}, \eta_{h}$ are expanded over each triangle $K$ of $T_{h}$ in the finite element basis such that

$$
\mathbf{u} \simeq \mathbf{u}_{h}=\sum_{p=1}^{N_{s}} \mathbf{u}_{p} \phi_{p}, \quad \eta \simeq \eta_{h}=\sum_{s=1}^{N_{v}} \eta_{s} \zeta_{s},
$$

for the formulation $\mathbf{F}_{1}$, and for the formulation $\mathbf{F}_{2}$, we obtain

$$
\mathbf{u} \simeq \mathbf{u}_{h}=\sum_{p=1}^{N_{s}} \mathbf{u}_{p} \varphi_{p}, \quad \eta \simeq \eta_{h}=\sum_{s=1}^{N_{v}} \eta_{s} \psi_{s},
$$

where $\eta_{s}$ and $\mathbf{u}_{p}$ represent the nodal values of surface elevation and velocity field respectively, while $\zeta_{s}, \psi_{s}$ and $\phi_{p}, \varphi_{p}$ are the basic functions of surface elevation and velocity components respectively. $N_{v}$ is the number of surface elevation nodes and $N_{s}$ is the number of velocity nodes in the mesh. 
Equations (6) -(7) lead to

$$
\begin{aligned}
\sum_{K \in T_{h}} \sum_{p \in I_{K}}\left[\alpha \mathbf{u}_{p}+f \mathbf{k} \times \mathbf{u}_{p}\right] \int_{K} \phi_{p} \boldsymbol{\phi}_{q} d \mathbf{x}-g \sum_{K \in T_{h}} \sum_{s \in K} \eta_{s} \int_{K} \zeta_{s} \boldsymbol{\nabla} \cdot \boldsymbol{\phi}_{q} d \mathbf{x}=0, \\
\sum_{K \in T_{h}} \sum_{s \in K} \alpha \eta_{s} \int_{K} \zeta_{s} \zeta_{r} d \mathbf{x}+\sum_{K \in T_{h}} \sum_{p \in I_{K}} \mathbf{u}_{p} \cdot \int_{K} \boldsymbol{\nabla} \cdot\left(H \phi_{p}\right) \zeta_{r} d \mathbf{x}=0,
\end{aligned}
$$

and equations (8) $-(9)$ yield

$$
\begin{aligned}
\sum_{K \in T_{h}} \sum_{p \in I_{K}}\left[\alpha \mathbf{u}_{p}+f \mathbf{k} \times \mathbf{u}_{p}\right] \int_{K} \varphi_{p} \boldsymbol{\varphi}_{q} d \mathbf{x}+g \sum_{K \in T_{h}} \sum_{s \in K} \eta_{s} \int_{K}\left(\nabla \psi_{s}\right) \cdot \boldsymbol{\varphi}_{q} d \mathbf{x}=0, \\
\sum_{K \in T_{h}} \sum_{s \in K} \alpha \eta_{s} \int_{K} \psi_{s} \psi_{r} d \mathbf{x}-\sum_{K \in T_{h}} \sum_{p \in I_{K}} \mathbf{u}_{p} \cdot \int_{K} H \varphi_{p}\left(\nabla \psi_{r}\right) d \mathbf{x}=0,
\end{aligned}
$$

where $\boldsymbol{\phi}_{p}=\left(\phi_{p}, \phi_{p}\right), \boldsymbol{\varphi}_{p}=\left(\varphi_{p}, \varphi_{p}\right), I_{K}$ is the set of velocity nodes belonging to $K$. Each system in (12) $-(15)$ can be rewritten as a generalized eigenvalues problem

$$
\left(\begin{array}{c||c}
\mathbf{C} & \mathbf{G} \\
\hline \hline \mathbf{D} & \mathbf{O}_{1}
\end{array}\right)\left(\begin{array}{c}
\mathbf{U} \\
\hline \overline{\boldsymbol{\eta}}
\end{array}\right)=\alpha\left(\begin{array}{c||c}
-\mathbf{M}^{u} & \mathbf{O}_{3} \\
\hline \hline \mathbf{O}_{2} & -\mathbf{M}^{\eta}
\end{array}\right)\left(\begin{array}{c}
\mathbf{U} \\
\hline \bar{\eta}
\end{array}\right)
$$

where $\mathbf{M}^{u}$ is the $N_{u} \times N_{u}$ mass matrix for $\mathbf{u}, \mathbf{M}^{\eta}$ is the $N_{\eta} \times N_{\eta}$ mass matrix for $\eta, \mathbf{D}$ is the $N_{\eta} \times N_{u}$ divergence matrix, $\mathbf{G}$ is the $N_{u} \times N_{\eta}$ gradient matrix, $\mathbf{O}_{1}$ is the $N_{\eta} \times N_{\eta}$ null matrix, $\mathbf{O}_{2}$ is the $N_{\eta} \times N_{u}$ null matrix, $\mathbf{O}_{3}$ is the $N_{u} \times N_{\eta}$ null matrix, $\mathbf{C}$ is the $N_{u} \times N_{u}$ Coriolis matrix and

$$
\mathbf{U}=\left(\begin{array}{llllll}
u_{1} & v_{1} & u_{2} & v_{2} & \ldots & u_{N_{u}} v_{N_{u}}
\end{array}\right)^{T}, \quad \boldsymbol{\eta}=\left(\begin{array}{lllll}
\eta_{1} & \eta_{2} & \ldots & \eta_{N_{\eta}}
\end{array}\right)^{T},
$$

with $N_{u}$ and $N_{\eta}$ being the number of velocity and surface elevation nodes, respectively.

\subsection{The Discontinuous Galerkin Method}

The time discretized equations (4)-(5) are considered and the discontinuous Galerkin method is now employed for the space discretization. Details can be found in ( Cokburn, 1997), ( Cokburn, 2003) and (Shipton J \& Gibson T.H. \& Cotter C.J., 2018).

In order to describe the weak formulation, a few notations are first defined. Let $\left\{T_{h}\right\}_{h>0}$ be a partition of the domain $\Omega$ into a finite number $N_{e l}$ of disjoint open elements $K_{e l}$ such that

$$
\bar{\Omega}=\cup_{i=1}^{N_{e l}} \bar{K}_{i} \text { and } \bar{K}_{i^{+}} \cap \bar{K}_{i^{-}}=\varnothing \text { for } i^{+} \neq i^{-},
$$

where $h$ is the maximum diameter of the element, $\bar{\Omega}$ is the adhesion of $\Omega$ and each element $K_{i}$ has a Lipschitzian boundary $\partial K_{i}$. The mesh length parameter $h$ is assumed to be constant. Let $\Gamma$ be the finite set of $M$ inter-element boundaries $\Gamma_{K_{i}}=\bar{K}_{i^{+}} \cap \bar{K}_{i^{-}}$with $i^{+}>i^{-}$within the domain, with all possible combinations

$$
\bar{\Gamma}=\cup_{i=1}^{M} \bar{\Gamma}_{K_{i}} \text { and } \bar{\Gamma}_{K_{i^{+}}} \cap \bar{\Gamma}_{K_{i^{+}}}=\varnothing \text { for } i^{+} \neq i^{-} .
$$

Each inter-element boundary $\Gamma_{K} \in \Gamma$ is associated with a unique fixed unit normal vector denoted by $\mathbf{n}_{K}=\left(n_{K}^{x}, n_{K}^{y}\right)$. Further, for any function $\zeta \in V_{K}:=H^{1}(K)$, where $\zeta$ represents $u, v$ or $\eta$, and for each element $K$, the trace of $\zeta$ on interior edges $\Gamma_{K}$ is denoted by $\zeta^{ \pm}$, or $\zeta_{L}$ ( $L$ for left) and $\zeta_{R}$ (R for right). For $\mathbf{x} \in \Gamma_{K}$ we have

$$
\zeta_{g}(\mathbf{x})=\zeta^{-}(\mathbf{x})=\lim _{\epsilon \rightarrow 0^{-}} \zeta\left(\mathbf{x}+\epsilon \mathbf{n}_{K}\right) \zeta_{d}(\mathbf{x})=\zeta^{+}(\mathbf{x})=\lim _{\epsilon \rightarrow 0^{+}} \zeta\left(\mathbf{x}+\epsilon \mathbf{n}_{K}\right) .
$$

To obtain the weak formulation both gradient term in (4) and divergence term in (5) are integrated by parts, which lead to the weak formulations $\mathbf{F 3}$ defined as follow.

Formulation F3: Let $V_{3}$ be a subspace of $H^{1}(K), \forall K \in T_{h}, \mathbf{u} \in V_{3} \times V_{3}$ and $\eta \in V_{3}$. The weak formulation is obtained by multiplying (4) by a test function $\phi(\mathbf{x})=(\phi(\mathbf{x}), \phi(\mathbf{x}))$, then multiplying (5) by a test function $\phi(\mathbf{x})$ with $\phi(\mathbf{x}) \in V_{3} \times V_{3}$ and $\phi(\mathbf{x}) \in V_{3}$, and integrating over the domain leads to 


$$
\begin{aligned}
\alpha \int_{K} \mathbf{u} . \boldsymbol{\phi} d \mathbf{x}+f \int_{K}(\mathbf{k} \times \mathbf{u}) \cdot \boldsymbol{\phi} d \mathbf{x}-g \int_{K} \eta \boldsymbol{\nabla} \cdot \boldsymbol{\phi} d \mathbf{x}+\int_{\partial K} g \eta \boldsymbol{\phi} . \mathbf{n}_{k} d s=0, \\
\alpha \int_{K} \eta \phi d \mathbf{x}-\int_{K} H \mathbf{u} \cdot \boldsymbol{\nabla} \phi d \mathbf{x}+\int_{\partial K} H \phi \mathbf{u} \cdot \mathbf{n}_{k} d s=0 .
\end{aligned}
$$

The Discontinuous Galerkin method approaches the solutions of (21)-(22) in a finite dimensional subspace. Let $V_{3, h}$ be the finite-dimensional subspaces of $V_{3}$. The solution $\mathbf{u}_{h}$ (resp. $\eta_{h}$ ) belongs to $V_{3, h} \times V_{3, h}$ (resp. $V_{3, h}$ ) defined as beeing the set of functions $\mathbf{u}_{h}$ (resp. $\eta_{h}$ ) whose restriction on a triangle $K$ of $T_{h}$ belongs to $P_{m_{1}}(K) \times P_{m_{1}}(K)$ (resp. $P_{m_{1}}(K)$ ), where $P_{m_{1}}(K)$ denotes the set of polynomials of degree $m_{1}$ defined on $K$.

The introduction of the finite element basis leads to a formulation as in (21)-(22), but with $\mathbf{u}, \eta$ replaced by the finite element trial functions $\mathbf{u}_{h}, \eta_{h}$ and $\phi$ replaced by the corresponding finite element test function $\phi_{h} \in V_{3, h} \times V_{3, h}, \phi_{h} \in V_{3, h}$.

Equations (21) -(22) lead to

$$
\begin{aligned}
& \alpha \sum_{K \in T_{h}} \int_{K} \mathbf{u}_{h} \cdot \boldsymbol{\phi}_{h} d \mathbf{x}+f \sum_{K \in T_{h}} \int_{K}\left(\mathbf{k} \times \mathbf{u}_{h}\right) \cdot \boldsymbol{\phi}_{h} d \mathbf{x}-g \sum_{K \in T_{h}} \int_{K} \eta_{h} \boldsymbol{\nabla} \cdot \boldsymbol{\phi}_{h} d \mathbf{x} \\
&+\sum_{K \in T_{h}} \int_{\partial K} g \eta^{*} \boldsymbol{\phi} \cdot \mathbf{n}_{K} d s=0, \\
& \alpha \sum_{K \in T_{h}} \int_{K} \eta_{h} \phi_{h} d \mathbf{x}-\sum_{K \in T_{h}} \int_{K} H \mathbf{u}_{h} \cdot \mathbf{\nabla} \phi_{h} d \mathbf{x}+\sum_{K \in T_{h}} \int_{\partial K} H \phi \mathbf{u}^{*} \cdot \mathbf{n}_{K} d s=0,
\end{aligned}
$$

where $\boldsymbol{\phi}_{h}=\left(\phi_{h}, \phi_{h}\right),\left(\mathbf{u}^{*}, \eta^{*}\right)$ denote the numerical trace of $(\mathbf{u}, \eta)$ on the boundary element $\partial K$ with $\mathbf{u}^{*}=\left(u^{*}, v^{*}\right)$. Note that the finite volume scheme is obtained from (23) - (24) when $m=0$, and hence the third term in the left hand side of (23) and the second term in the left hand side of (24) are all zeros as the test function belongs to $P_{0}(K)$. To complete the definition of the approximate solution $\left(u_{h}, v_{h}, \eta_{h}\right)$, it only remains to choose a unique numerical flux $\left(u^{*}, v^{*}, \eta^{*}\right)$ at the cell interface as to render the method consistent and stable. By using the Rusanov flux which guarentees the stability, we obtain

$$
\left(\begin{array}{c}
g \eta^{*} \mathbf{n}_{K} \\
H \mathbf{u}^{*} \cdot \mathbf{n}_{K}
\end{array}\right)=\left(\begin{array}{c}
g \mathbf{n}_{K}\{\eta\}-\frac{\sqrt{g H}}{2}[\mathbf{u}] \\
H \mathbf{n}_{K} \cdot\{\mathbf{u}\}-\frac{\sqrt{g H}}{2}[\eta]
\end{array}\right),
$$

with $\zeta \in\{u, v, \eta\},[\zeta]=\zeta_{R}-\zeta_{L}$ is the jump of $\zeta$ and $\{\zeta\}=\frac{\zeta_{R}+\zeta_{L}}{2}$ is the mean of $\zeta$. After expanding $\mathbf{u}$ and $\eta$ on each triangle $K$ of $T_{h}$, in terms of their respective nodal values and basis functions, we obtain the generalized eigenvalue problem

$$
\left(\begin{array}{c||c}
\mathbf{C}+\mathbf{S}^{u} & \mathbf{G} \\
\hline \mathbf{D} & \mathbf{S}^{\eta}
\end{array}\right)\left(\begin{array}{c}
\mathbf{U} \\
\hline \overline{\boldsymbol{\eta}}
\end{array}\right)=\alpha\left(\begin{array}{c||c}
-\mathbf{M}^{u} & \mathbf{O} \\
\hline \hline \mathbf{O} & -\mathbf{M}^{\eta}
\end{array}\right)\left(\begin{array}{c}
\mathbf{U} \\
\hline \boldsymbol{\eta}
\end{array}\right),
$$

where $\mathbf{M}^{u}$ is the $2 N \times 2 N$ mass matrix for $\mathbf{u}, \mathbf{M}^{\eta}$ is the $N \times N$ mass matrix for $\eta, \mathbf{D}$ is the $N \times 2 N$ divergence matrix, $\mathbf{G}$ is the $2 N \times N$ gradient matrix, $\mathbf{O}$ is the null matrix, $\mathbf{C}$ is the $2 N \times 2 N$ Coriolis matrix, $\mathbf{S}^{u}$ is the $2 N \times 2 N$ stabilization matrix for $\mathbf{u}, \mathbf{S}^{\eta}$ is the $N \times N$ stabilization matrix for $\eta$ and

$$
\mathbf{U}=\left(\begin{array}{lllllll}
u_{1} & v_{1} & u_{2} & v_{2} & \ldots & u_{N} & v_{N}
\end{array}\right)^{T}, \quad \boldsymbol{\eta}=\left(\begin{array}{llll}
\eta_{1} & \eta_{2} & \ldots & \eta_{N}
\end{array}\right)^{T} .
$$

Note that $\mathbf{S}^{u}$ and $\mathbf{S}^{\eta}$ are the stabilization matrices regrouping the jumps of $\mathbf{u}$ and $\eta$, respectively.

\section{Numerical Results}

All numerical results are obtained using FreeFem++ of (Hecht, F , 2012). Equations (12) -(15) are solved in a rectangular domain of length $L_{x}$ and width $L_{y}$ on a structured mesh, the Coriolis force $f$ being constant. The boundary of the domain is subject to periodic conditions.

This test is performed using finite element pairs $P_{0}-P_{1}, P_{1}^{D G}-P_{1}, P_{1}^{D G}-P_{2}, P_{1}^{N C}-P_{1}, P_{2}-P_{1}$, and $P_{1}^{D G}-P_{1}^{D G}$. The structured mesh is composed of right isosceles triangles, and $a$ and $b$ denote the number of segments on the length and on the width of the domain, respectively. Several variable bathymetries have been tested and all of them lead to the same conclusions. We vary the values of $a$ and $b$ and in each case we compare the number of eigenvalues of (16) and (26) with 
the product $a b$. The results presented in this study have been obtained with three variable bathymetries $H_{1}, H_{2}$ and $H_{3}$ defined by (28), (29) and (30) respectively, and shown in Figure 1 . The bathymetry $H_{1}$ is defined by

$$
H_{1}(x, y)=H_{0}\left(1-d \cdot e^{-c\left(\left(x-x_{0}\right)^{2}+\left(y-y_{0}\right)^{2}\right)}\right),
$$

where $H_{0}$ is the depth around which the pertubation takes place, and $c$ and $d$ well chosen constants, with $c=0.02$ and $d=0.003$, and $\left(x_{0}, y_{0}\right)$ is the origin of the domain $\Omega$. The bathymetry $H_{2}$ on Figure 1 is defined by

$$
H_{2}(x, y)=H_{0}\left(1+d \cdot e^{-c\left(\left(x-x_{0}\right)^{2}+\left(y-y_{0}\right)^{2}\right)}\right),
$$

while the bathymetry $H_{3}$ reads

$$
H_{3}(x, y)=\left\{\begin{array}{l}
1.63, \quad \forall(x, y) \in[-2.39,-1.195] \times[-1.43,1.43] \\
0.3 x+1.99, \quad \forall(x, y) \in[-1.195,1.195] \times[-1.43,1.43] \\
2.3485, \quad \forall(x, y) \in[1.195,2.39] \times[-1.43,1.43]
\end{array}\right.
$$
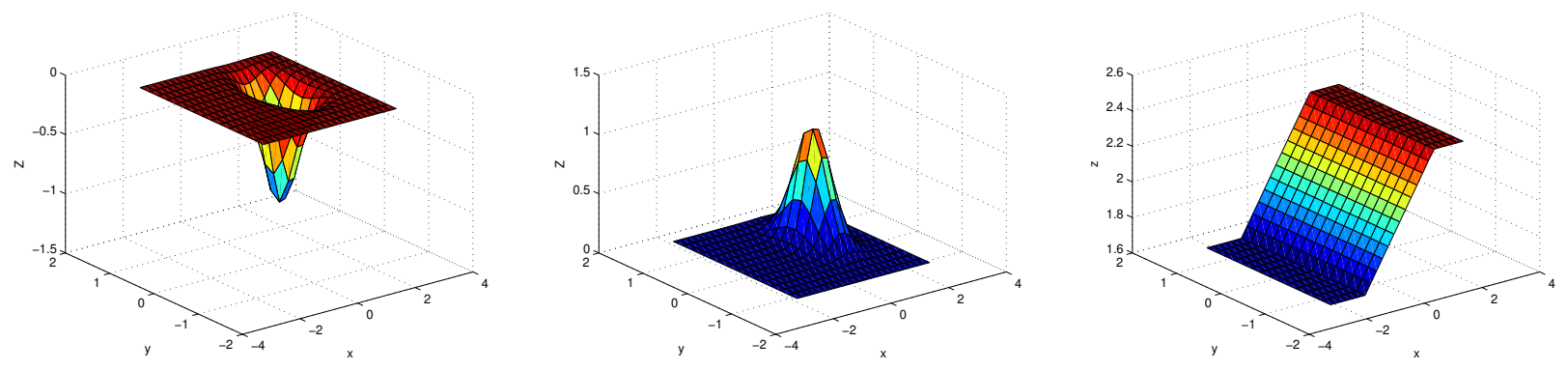

Figure 1 . The bathymetries: (left) $H_{1}$, (center) $H_{2}$ and (right) $H_{3}$

At initial time, an anti-cyclonic velocity field is in geostrophic equilibrium with a prescribed Gaussian distribution of the surface elevation, centered at the origin of the domain. This leads to the following initial conditions

$$
\begin{aligned}
\widetilde{\eta}(x, y, t=0) & =\kappa e^{-\sigma\left(\left(x-x_{0}\right)^{2}+\left(y-y_{0}\right)^{2}\right)}, \\
\widetilde{\mathbf{u}}(x, y, t=0) & =\frac{g}{f} \mathbf{k} \times \nabla \widetilde{\eta}(x, y, t=0),
\end{aligned}
$$

with $\kappa$ and $\sigma$ are well chosen constants, for example $\kappa=0.02$ and $\sigma=0.003$. In the model, the equations were adimentionalized with the inertial period $T=104504 s$, the characteristic time. When computing the eigenvalues of (16), we obtained three kind of values: some values equal to 0 , some values equal to \pm 7.6178 and the others are such that their absolute value is greater than 7.6178. To get the dimentionalized value, we divide each pulsation value by $T$. Indeed we have $E=e^{i \omega \Delta t}, e^{i \omega \Delta t}=e^{i \omega^{\prime} \Delta t^{\prime}}$ and $e^{i \omega \Delta t}=e^{i \omega T \Delta t^{\prime}}$. So the numerical value $\omega^{\prime}$ computed is such that $\omega^{\prime}=\omega T$. Thus $7.6178 / T \simeq 7.2894 \times 10^{-5}$ and this correspond to $f=2 \Omega_{e} \sin (\Phi)=\Omega_{e}=7.2921 \times 10^{-5} \mathrm{rad}_{\text {. }}{ }^{-1}$ for $\Phi=30^{\circ}$. The parameter $\Phi$ is equal to $30^{\circ}$ in the simulation. This implies the following remark.

Remark 1. In the numerical results the values $f= \pm 7.6178$ correspond to the spurious inertial modes, the values 0 correspond to the geostrophic modes and the others values such that their absolute value is greater than 7.6178 correspond to the inertia-gravity modes. We set $f_{s p}=7.6178$.

We vary the values of $a$ and $b$ in the range of integers $\llbracket 2 ; 12 \rrbracket$. In each case we compare the number of geostrophic modes $\omega=0$, inertial modes $\omega= \pm f$ and inertia-gravity modes corresponding to values of $\omega$ such that $|\omega|>f_{s p}$ with the product $a b$.

In the continuous case we have one geostrophic mode $\omega=0$, zero spurious inertial mode $\omega= \pm f$ and 2 inertia-gravity modes $\omega= \pm \sqrt{f^{2}+g H\left(k^{2}+l^{2}\right)}$, where $k$ and $l$ are the wave numbers in the $\mathrm{x}$ - and $\mathrm{y}$-directions, respectively. We denote by $G M$ the geostrophic modes, IGM the inertia-gravity modes, $S I M$ the spurious inertial modes, $F E$ the finite element and $R S I M$ the ratio of spurious inertial modes, namely $R S I M=\frac{N_{S I M}}{N_{G M}+N_{S I M}+N_{I G M}}$, where $N_{S I M}, N_{G M}$ and $N_{I G M}$ 
are the number of spurious inertial modes, the number of geostrophic modes and the number of inertia-gravity modes respectively.

In order to determine the influence of bathymetry on the existence and proportions of the spurious inertial modes, we firstly compute the number of modes when the bathymetry is constant. We vary the value of $(a, b)$ in any $(a, b) \in \llbracket 2 ; 12 \rrbracket^{2}$ and results are shown in Table 1.

Table 1. Results of eigenvalues computed with a constant bathymetry for the $P_{2}-P_{1}, P_{1}^{N C}-P_{1}, P_{0}-P_{1}, P_{1}^{D G}-P_{1}$, $P_{1}^{D G}-P_{2}, P_{1}^{D G}-P_{1}^{D G}$, finite element pairs, for any $(a, b) \in \llbracket 2 ; 12 \rrbracket^{2}$

\begin{tabular}{|c|c|c|c|c|c|c|c|c|c|c|c|c|}
\hline \multirow{2}{*}{$\begin{array}{l}\text { Mode } \\
\text { type }\end{array}$} & \multicolumn{2}{|c|}{$P_{2}-P_{1}$} & \multirow{2}{*}{\multicolumn{2}{|c|}{\begin{tabular}{l|l}
$P_{1}^{N C}-P_{1}$ \\
$\omega^{N}$ & number
\end{tabular}}} & \multicolumn{2}{|c|}{$P_{0}-P_{1}$} & \multicolumn{2}{|c|}{$P_{1}^{D G}-P_{1}$} & \multicolumn{2}{|c|}{$P_{1}^{D G}-P_{2}$} & \multicolumn{2}{|c|}{$P_{1}^{D G}-P_{1}^{D G}$} \\
\hline & $\omega$ & number & & & $\omega$ & number & $\omega$ & number & $\omega$ & number & $\omega^{1}$ & number \\
\hline GM & 0 & $a b$ & 0 & $a b$ & 0 & $a b$ & 0 & $a b$ & 0 & $4 a b$ & 0 & $6 a b$ \\
\hline SIM & $\pm f_{s p}$ & $6 a b$ & $\pm f_{s p}$ & $4 \mathrm{ab}$ & $\pm f_{s p}$ & $2 \mathrm{ab}$ & $\pm f_{s p}$ & $10 \mathrm{ab}$ & $\pm f_{s p}$ & $4 a b$ & $\pm f_{s p}$ & 0 \\
\hline IGM & $\omega^{g}$ & $2 a b$ & $\omega^{g}$ & $2 \mathrm{ab}$ & $\omega^{g}$ & $2 a b$ & $\omega^{g}$ & $2 a b$ & $\omega^{g}$ & $8 \mathrm{ab}$ & $\omega^{g}$ & $12 \mathrm{ab}$ \\
\hline RSIM & & $6 / 9$ & & $4 / 7$ & & $2 / 5$ & & $10 / 13$ & & $4 / 16$ & & 0 \\
\hline
\end{tabular}

Note: $\omega^{g}$ any pulsation $\omega$ such that $|\omega|>f_{s p}$.

For all variable bathymetries considered in this study we obtained the same values and numbers of spurious inertial and geostrophic modes. However, if the number of inertia-gravity modes is the same, their value changes only slightly, from one bathymetry to another. For example, when taking $(a, b)=(5 ; 3)$ we obtain as maximum mode values $8.8942,8.8943$ and 8.8944 for bathymetries $H_{1}, H_{2}$ and $H_{3}$ respectively.

Table 2. The geostrophic, spurious inertial and inertia-gravities modes proportions for the $P_{2}-P_{1}, P_{1}^{N C}-P_{1}, P_{0}-P_{1}$, $P_{1}^{D G}-P_{1}, P_{1}^{D G}-P_{2}, P_{1}^{D G}-P_{1}^{D G}$ finite element pairs, for any $(a, b) \in \llbracket 2 ; 12 \rrbracket^{2}$ with variable bathymetries

\begin{tabular}{l|c|c|c|c|c|c|c}
\hline Modes & Continuous & $P_{2}-P_{1}$ & $P_{1}^{N C}-P_{1}$ & $P_{0}-P_{1}$ & $P_{1}^{D G}-P_{1}$ & $P_{1}^{D G}-P_{2}$ & $P_{1}^{D G}-P_{1}^{D G}$ \\
\hline GM & 0 & $a b$ & $a b$ & $a b$ & $a b$ & $4 a b$ & $6 a b$ \\
\hline SIM & $\pm f_{s p}$ & $6 a b$ & $4 a b$ & $2 a b$ & $10 a b$ & $4 a b$ & 0 \\
\hline IGM & $|\omega|>f_{s p}$ & $2 a b$ & $2 a b$ & $2 a b$ & $2 a b$ & $8 a b$ & $12 a b$ \\
\hline RSIM & & $6 / 9$ & $4 / 7$ & $2 / 5$ & $10 / 13$ & $4 / 16$ & 0 \\
\hline
\end{tabular}

Further, when using $(a, b)=(5 ; 3)$ we obtain $90,60,30,150,60,0$ values equal to $\pm f_{s p}$, namely $f= \pm 7.6178$, corresponding to the number of spurious inertial modes for the finite element pairs $P_{2}-P_{1}, P_{1}^{N C}-P_{1}, P_{0}-P_{1}, P_{1}^{D G}-P_{1}$, $P_{1}^{D G}-P_{2}, P_{1}^{D G}-P_{1}^{D G}$, respectively.

As a conclusion, the mode proportion results obtained in this section are consistent with those of Table 5 of (Le ROUX, 2012) for all finite element pairs that have two velocity components per node considered in this study. The physical parameters that are employed to discretize the SW equations do not affect the results in these tables for any $(a, b) \in$ $\llbracket 2 ; 12 \rrbracket^{2}$. Thus, the results obtained in Table 5 of (Le ROUX, 2012) in the case of a constant bathymetry are the same as for the variable bathymetries used in this study. A regular variation of the bathymetry does not change the proportions of the modes.

We compute the eigenvalues of (26) using the Rusanov flux and we noted that a finite element space discretization using the discontinuous Galerkin method does not reveal any spurious inertial mode for constant and variable bathymetries. This is explained by the fact that the approximation spaces of the velocity field and the elevation of the free surface elevation are the same. Thus the principal responsable of spurious modes seems to be the interaction of different approximation spaces of the finite element pairs that are used.

\section{Conclusion}

The study of the generalized eigenvalue problems presented in this paper allowed us to evaluate the proportions of the modes resulting from the discretization of the shallow water equations. The results obtained in section 4 , show that the regular variation of the bathymetry does not influence the number of spurious inertial modes. We therefore retain that in the case of a constant bathymetry and in the case of bathymetries with regular variations, the proportions of the inertial spurious modes for the $P_{2}-P_{1}, P_{1}^{N C}-P_{1}, P_{1}^{D G}-P_{1}, P_{1}^{D G}-P_{2}$ and $P_{0}-P_{1}$ finite elements pairs are $2 / 3,4 / 7,10 / 13,1 / 4,2 / 5$, respectively. This study then makes it possible to generalize some results of Table 5 of (Le ROUX, 2012) to the case of bathymetries with regular variations. 
The numerical results obtained from the discrete linear equations resulting from the formulations $\mathbf{F 1}$ and $\mathbf{F} 2$ show that the interaction between the approximation spaces of velocity field and the free surface elevation is the real cause of the appearance of spurious inertial modes. This is why in order to eliminate these spurious inertial modes we carry out a discretization in space of the 2D shallow water equations by using the discontinuous Galerkin method that approaches the velocity field and the free surface elevation in the same approximation space. We then determine the eigenvalues and the eigenvectors of the discrete linear system and we analyze these eigenvalues that correspond to the different modes of the system and we observe the absence of spurious inertial modes.

\section{References}

Adou, J. K., Sitionon, G., \& Coulibaly, A. (2016). Numerical study of the flow in a channel located close to a punctual singular vortex. Advances and Applications in Fluid Mechanics, 19(2), 415-426. http://dx.doi.org/10.17654/FM019020415

Cockburn, B. (1997). An introduction to the discontinuous Galerkin method for convection-domainated problem School of Mathematics. University of Minnesota, Minneapolis, Minnesota, 55455, USA. https://doi.org/10.1007/BFb0096353

Cockburn, B. (2003). Discontinuous Galerkin Methods. School of Mathematics Univeristy of Minnesota, 1-25. https://doi.org/10.1002/zamm.200310088

Eldred, C., \& Le Roux, D. Y. (2018). Dispersion analysis of compatible Galerkin schemes for the 1D shallow water model. Journal of Computational Physics, 371, 779-800. https://doi.org/10.1016/j.jcp.2018.06.007

Eldred, C., \& Le Roux, D. Y. (2019). Dispersion analysis of compatible Galerkin schemes on quadrilaterals for shallow water models. Journal of Computational Physics, 387, 539-568. https://doi.org/10.1016/j.jcp.2019.02.009

Hecht, F. (2012). New development in FreeFem++. Journal of numerical mathematics, 20(3-4), 251-266. https://doi.org/10.1515/jnum-2012-0013.

Le Roux, D. Y. (2012). Spurious inertial oscillations in shallaw-water models. Journal of Computational Physics, 231, 7959-7987. http://dx.doi.org/10.1016/j.jcp.2012.04.052

Shipton, J., Gibson, T. H., \& Cotter, C. J. (2018). Higher-order compatible finite element schemesfor the nonlinear rotating shallow water equations on the sphere. Journal of Computational Physics, 375, 1121-1137. https://doi.org/10.1016/j.jcp.2018.08.027

\section{Copyrights}

Copyright for this article is retained by the author(s), with first publication rights granted to the journal.

This is an open-access article distributed under the terms and conditions of the Creative Commons Attribution license (http://creativecommons.org/licenses/by/4.0/). 